\title{
Electron-beam-induced-current and active secondary-electron voltage- contrast with aberration-corrected electron probes
}

Myung-Geun Han ${ }^{\mathrm{a},}$, Joseph A. Garlow ${ }^{\mathrm{a}, \mathrm{b}}$, Matthew S. J. Marshall ${ }^{\mathrm{c}}$, Amanda L. Tiano ${ }^{\mathrm{c}}$, Stanislaus S. Wong ${ }^{\text {a,c }}$, Sang-Wook Cheong ${ }^{\text {, }}$, Frederick J. Walker ${ }^{\text {e,f }}$, Charles H. Ahn ${ }^{\text {e,f }}$, and Yimei $\mathrm{Zhu}^{\mathrm{a}}$

${ }^{a}$ Condensed Matter Physics \& Materials Science, Brookhaven National Laboratory, Upton, NY, USA 11973

${ }^{\mathrm{b}}$ Materials Science and Engineering Department, State University of New York at Stony Brook, Stony Brook, NY, USA 11794

${ }^{\mathrm{c}}$ Department of Chemistry, State University of New York at Stony Brook, Stony Brook, NY, USA 11794-3400

${ }^{\mathrm{d}}$ Department of Physics and Astronomy, Rutgers Center for Emergent Materials, Rutgers

University, Piscataway, NJ, USA 08854

${ }^{\mathrm{e}}$ Department of Applied Physics and Center for Research on Interface Structures and Phenomena, Yale University, New Haven, CT, USA 06520

${ }^{\mathrm{f}}$ Department of Mechanical Engineering and Materials Science, Yale University, New Haven, CT, USA 06520

Abstract

The ability to map out electrostatic potentials in materials is critical for the development and the design of nanoscale electronic and spintronic devices in modern industry. Electron holography has been an important tool for revealing electric and magnetic field distributions in microelectronics and magnetic-based memory devices, however, its utility is hindered by several practical constraints, such as charging artifacts and limitations in sensitivity and in field of view. In this article, we report electron-beaminduced-current (EBIC) and secondary-electron voltage-contrast (SE-VC) with an aberration-corrected electron probe in a transmission electron microscope (TEM), as complementary techniques to electron holography, to measure electric fields and surface potentials, respectively. These two techniques were applied to ferroelectric thin films, multiferroic nanowires, and single crystals. Electrostatic potential maps obtained by offaxis electron holography were compared with EBIC and SE-VC to show that these 
techniques can be used as a complementary approach to validate quantitative results obtained from electron holography analysis.

Nanoscale control of electric fields in modern electronic devices is a prerequisite to improving their functionalities in conjunction with the scaling demand for high-density semiconductor devices. One of the key factors is interfaces/junctions, where built-in electric fields are generally predicted. At thermal equilibrium, the necessity of Fermi level equilibration across interfaces/junctions requires charge exchange, leaving net space charges on either one or both sides of the interface. These space charges yield built-in electric fields that prevent further charge exchange across the interface/junction to reach a steady state. Experimental measurements of these built-in electric fields are of great importance to predict and control charge transport properties in advanced devices that involve photovoltaic effects, rectifications, or capacitance modulations, etc.

In electron microscopy, off-axis electron holography has been an important tool for mapping 2D electric fields in sub-micrometer semiconductor field-effect transistors, semiconductor nanowires, and nanocrystals, etc. [1-5]. However, practical limitations in addition to the stringent requirements of TEM sample preparation limit the efficacy of off-axis electron holography. When the sample is charging, the reference wave coming from vacuum in proximity to the area of interest is disturbed, thereby preventing reliable quantitative analysis [6]. In addition, requirements on the lens conditions limit the available field of views and spatial resolutions [1].

Meanwhile, both EBIC and SE-VC have been widely utilized to study correlations between electronic properties and structural defects as well as failure 
analysis in semiconductor devices [7-10]. These two techniques have been mainly performed in a scanning electron microscope (SEM), however, there are also numerous studies that have been performed in a scanning transmission electron microscope (STEM) [11-14]. The difficulty here is that electrical connection to a TEM sample is a challenging task. When $200 \mathrm{keV}$ electrons are passing through a TEM sample, they generate electron hole pairs (ehps) and secondary electrons via inelastic scattering processes. Previously, we have shown that each $200 \mathrm{keV}$ electron can generate $\sim 100$ electron hole pairs in crystalline $\mathrm{Si}$ [15]. The ehps are excess charge carriers, which eventually recombine to annihilate each other with a characteristic time scale, called excess carrier lifetime. During this lifetime, these carriers may diffuse in random directions before recombination. When semiconductors are doped, either $n$-type (electrons being a majority charge carrier) or $p$-type (holes being a majority charge carrier), the ehps lifetime is determined by the minority carrier lifetime because there are so many majority carriers that are available for recombination.

When there is an electric field, either externally applied or built-in, as in the depletion region at either a $p-n$ junction or a Schottky junction, electrons and holes move in opposite directions and, thus, generate a current (EBIC) in the direction of the field. This induced current for each scanning electron beam synchronized with STEM coils is measured and used as the imaging signal to acquire an EBIC image. As a result, the EBIC intensity is proportional to the magnitude of local electric fields for a given excitation. Commercial SEM-based systems afford a minimum current down to $0.8 \mathrm{fA}$ using a lockin technique [16]. The minimum measurable current in this study is in the range of a few tens of pico-Amperes without lock-in filtering. 
SE-VC has been extensively utilized, especially to localize failure sites in integrated semiconductor circuits. Generally, different intensities in SEM images help to identify electrical shorts and/or disconnects in circuits. For this "passive" VC case, it is not necessary to apply an external bias. However, the intensity differences can be more pronounced if an external bias is applied to the circuit under test. This is because the secondary electron yield is not only sensitive to the incoming electron energy, but also to local electrostatic potentials for a given material. When a positive (negative) external bias is applied to the material, the secondary electron yield is suppressed (enhanced) by simple electrostatic interactions. This "active" VC method can detect the failures occurring in a device under operating conditions.

A key advantage of EBIC and SE-VC with thin TEM samples is that the interaction volume is small due to reduced electron beam broadening. Therefore, the spatial resolution is not degraded by SEs generated by backscattered electrons (called "SE2") but is mainly determined by SEs generated by the primary imaging electrons ("SE1"). This means that the spatial resolution of SEM on TEM samples is comparable to the probe size. With aberration-corrected electron probes, indeed atomic resolution has previously been demonstrated $[17,18]$. Another advantage of TEM samples is that the sample thickness is comparable to the escape depth of SE emission from both the top and bottom surfaces of the TEM sample. Ehp recombination at the top and bottom surface regions is also quite fast as compared with bulk and the surfaces likely serve as recombination centers. It should be noted that SE-VC imaging is sensitive to the sample surface, thus, the potential probed by SE-VC may be different from potentials for the bulk. 
In this article, we present electron-beam-induced-current (EBIC) measurement and secondary-electron voltage-contrast (SE-VC) obtained in STEM mode combined with in situ electrical biasing in a TEM. These techniques were applied to ferroelectric thin films, nanowires, and single crystals. A comparison with off-axis electron holography data demonstrates that although EBIC and SE-VC data are currently qualitative, they can be used as complementary measurements to support quantitative data obtained by off-axis electron holography.

We have used double aberration-corrected JEOL ARM 200 CF with cold fieldemission gun. The electrical connection to TEM samples for EBIC and SE-VC was made using a commercial TEM holder (Nanofactory Instruments). The probe current density was about $0.22 \mathrm{nA} / \mathrm{nm}^{2}$ for both EBIC and SE-VC experiments. EBIC signals were acquired simultaneously with STEM-HAADF images obtained with acceptance angles ranging from 68 to $280 \mathrm{mrad}$. Fig. 1 shows a schematic of EBIC and SE-VC measurements in STEM mode. The horizontal stripes in the EBIC image shown in Fig. 1 are artifacts that arise during EBIC signal acquisition and can be removed by adjusting signal amplification in the data collecting electronics (Gatan Digiscan II). We have used a JEOL backscattered electron detector for SE-VC imaging. The detectors are composed of two segments and collect not only SE, but also backscattered electrons, however, most of electrons detected are primarily SEs (85\%). As EBIC and SE-VC detectors are not interfering with HAADF and BF STEM detectors, all four images can be simultaneously acquired.

We have prepared a FIB-milled TEM sample of a $\mathrm{PbZr}_{0.2} \mathrm{Ti}_{0.8} \mathrm{O}_{3}$ (PZT) thin film grown onto conducting Nb-doped (001) $\mathrm{SrTiO}_{3}$ (Nb-STO) substrates, as shown in Fig. 
2(a). The sample is divided into two parts using a FIB cut (v-shape cut in the center part). One section on the left hand side in Fig. 2(a) is in the 'open-circuit' configuration as the top electrode is floated while the bottom electrode (conducting Nb-STO) is connected to the voltage source. The other section on the right is in the 'closed-circuit' configuration as the top and bottom electrodes are electrically connected each other by a molybdenum TEM grid (Omniprobe, Inc.). An electric contact was made to the open-circuited section with a tungsten probe using a commercial Nanofactory holder. Previously, we have shown that the PZT/Nb-STO interface behaves like a $p$ - $n$ junction, preventing polarization from switching at the interface when the polarization direction is parallel to the interfacial built-in electric field [19]. In Fig. 2(c) and (e), EBIC images taken from the two sections of the PZT/Nb-STO interface are shown together with HAADF images that were simultaneously acquired. A positive $2 \mathrm{~V}$ potential was applied to the $\mathrm{Nb}-\mathrm{STO}$ substrate while the tungsten probe was grounded during the image acquisition. The EBIC signal is clearly seen at the interface of PZT/Nb-STO in the open-circuit configuration (Fig. 2(c)), while the EBIC signal was not observed for the 'closed-circuit' configuration (Fig. 2(e)). In Fig. 2(f), the line profiles of EBIC signals are plotted as a function of applied bias to the Nb-STO substrate are shown. As a small positive voltage is applied to the $n$-type $\mathrm{Nb}$-STO, the PZT/Nb-STO is reversely biased. As a result, the depletion region across the interface is expanded to give larger EBIC signals. In contrast, for the 'closed-circuit' section, although there is a small built-in electric field across the PZT/Nb-STO interface to separate ehps, EBIC was not observed, as shown in Fig. 2(e). These results demonstrate that EBIC is not an artifact of STEM imaging and requires proper electrical connection to the TEM samples. As discussed previously, the EBIC 
signal can broaden due to ehp diffusion before collection as a current. By comparing Holography data with EBIC data shown in Fig 2(f) and 2(g), respectively, we have found that the ehp diffusion length for FIB-milled STO can be roughly estimated as $\sim 300 \mathrm{~nm}$ (for positive $2 \mathrm{~V}$, about $300 \mathrm{~nm}$ depletion region by EBIC and for positive $3 \mathrm{~V}, 50 \mathrm{~nm}$ by EH). Finally, it is interesting to note that the depletion region in PZT is fixed around 30 $\mathrm{nm}$ and narrower than that on the STO side. This may be associated with spontaneous polarization, charged defects (oxygen/cation vacancies and cation impurities) and their electric dipoles in the PZT film, which may limit the expansion of the PZT depletion region.

Active SE-VC has been performed on the same sample, as shown in Fig. 3 (b-d). The brightness of image changes depending upon the external bias applied to the NbSTO substrate with respect to the grounded top electrode. For example, when a positive 4 $\mathrm{V}$ potential is applied, the brightness of Nb-STO substrate is decreased with respect to the top electrode. On the other hand, when a negative $3 \mathrm{~V}$ potential is applied, the signal brightness of Nb-STO is enhanced. The brightness is also sensitive to the surface conditions of the TEM sample. When a sample is slightly contaminated by mainly hydrocarbons during extensive imaging, we have observed the SE image brightness is reduced. In Fig. 3(d), there is a rectangular area with suppressed contrast in the Nb-STO substrate due to surface contamination by extensive electron beam scanning. When there is no significant surface contamination, SE-VC is inversely proportional to the local electrostatic potential in the sample, because the SE yield will be suppressed (enhanced) with positive (negative) potentials. The inverted line profiles taken across the top electrode/PZT/Nb-STO in Fig. 3(e) show that SEM intensity is dramatically dependent 
upon external biases. It can be seen that a potential barrier develops at the PZT/Nb-STO interface as positive biases are applied to the $\mathrm{Nb-STO}$ substrate, which is consistent with a reverse-biased $p-n$ junction scenario [19]. However, compared to the line profiles of electrostatic potentials obtained by electron holography (Fig. 3(f)), more pronounced changes in the gradient, especially within the PZT film, are observed from the SEM data. This discrepancy can be explained by the effect of FIB-damaged surface layers, which may be considered as a linear resistor. Since SEM is more sensitive to surface conditions of sample than electron holography, surface electrostatic potentials in FIB-damaged layers can mask expected electrostatic potentials within TEM samples.

When forward (negative) biases are applied, the interface becomes conducting without noticeable potential barriers. The potential of the top electrode $(\mathrm{Pt} / \mathrm{Au})$ and bottom electrode (Nb-STO) are relatively flat and is either monotonously raised or lowered by external biases, as would be expected for good metals. Very dramatic changes in SE-VC are observed in the ferroelectric PZT film. In particular, the sign of the lineprofile slope is reversed as the polarity of the bias is correspondingly changed, in agreement with the change in the electric field direction. Although SE-VC results are qualitatively consistent with off-axis electron holography data, it is challenging to quantify SE-VC data. In addition, surface contamination during imaging should be addressed.

In Fig. 4, we have performed SE-VC on $\mathrm{YMn}_{2} \mathrm{O}_{5}$ nanowires synthesized by the molten salt method [20]. Both ends of the $\mathrm{YMn}_{2} \mathrm{O}_{5}$ nanowire are contacted - on one end to a $\mathrm{W}$ probe and to the $\mathrm{Cu}$ grid using hydrocarbon contamination accumulating under prolonged electron beam irradiation on the other end. The nanowire was about 3 um in 
length and $50 \mathrm{~nm}$ in diameter. Then, $+/-10 \mathrm{~V}$ external biases were applied to the $\mathrm{Cu}$ gird with respect to the $\mathrm{W}$ probe. A variation in SE-VC contrast with external biases is shown in line profiles taken along the long axis of the nanowire. There is a clear trend of the potential dependence with the observed SE brightness, similar to what was observed for the PZT sample shown in Fig. 3. At $0 \mathrm{~V}$, the area near the $\mathrm{W}$ probe is brighter than that near the $\mathrm{Cu}$ grid. For an insulating dielectric like $\mathrm{YMn}_{2} \mathrm{O}_{5}$, it is known that electrical grounding enhances SE brightness by sufficiently supplying charges lost during SE emission, a scenario which is widely observed in passive VC. On the other hand, there is no significant enhancement of brightness near the $\mathrm{Cu}$ grid. With a positive (negative) 10 $\mathrm{V}$ applied to the $\mathrm{Cu}$ grid, the brightness near the $\mathrm{Cu}$ grid is further reduced (enhanced) by the positive (negative) electrostatic potential. In contrast, the brightness of the $\mathrm{W}$ probe side is almost constant, therefore, most changes are observed near the $\mathrm{Cu}$ grid side where external biases were applied.

To estimate the effect of surface conditions, we have compared atomic-resolution SEM images obtained from FIB-milled $\mathrm{SrTiO}_{3}$ crystal and chemically synthesized ferroelectric $\mathrm{YMnO}_{3}$ nanowires. No FIB has been performed for $\mathrm{YMnO}_{3}$ nanowires while $\mathrm{SrTiO}_{3}$ single crystals were FIB-milled with $5 \mathrm{keV} \mathrm{Ga}$ ion as final milling. Both images highlight SEM atomic resolution in Fig. 5, although the signal to noise ratio is severely degraded for the $\mathrm{SrTiO}_{3}$ case due to FIB damage on sample surface. Meanwhile, the $\mathrm{YMnO}_{3}$ nanowire shows atomic resolution SE images comparable to the HAADF image. Hexagonal $\mathrm{YMnO}_{3}$ is ferroelectric at room temperature, and shows a spontaneous polarization that can be directly imaged by large Y-displacements along the $c$-axis. 
In single crystalline $\mathrm{YMnO}_{3}$, the Y-displacement patterns with up-up-down or down-down-up patterns along the [120] direction were directly imaged when viewed along the $a$-axis. In HAADF and SEM images shown in Fig. 5(a-b), however, the Ydisplacements are rather irregular, likely due to finite size effects. Here, the diameter of the $\mathrm{YMnO}_{3}$ nanowire is about $10 \mathrm{~nm}$ (the inset in Fig. 5(a)). Since Y-displacements are induced by antiferromagnetic trimerization of $\mathrm{Mn}^{3+}$ ions in the $a b$ plane, this irregular Ydisplacement may indicate severe disruptions in the antiferromagnetism, which could be of great interest for the coexistence of ferroelectric and ferromagnetic phases in $\mathrm{YMnO}_{3}$ nanowires.

For the case of the FIB-milled $\mathrm{SrTiO}_{3}$ sample, the FIB damage layer on both top and bottom sample surfaces provides a constant background, thereby reducing the signalto-noise ratio in the atomic resolution SEM image shown in Fig. 5(d). However, both $\mathrm{Sr}$ and Ti columns are clearly resolved (while the $\mathrm{O}$ columns are missing), implying efficient SE emission through FIB damage layers. The escape depth of SEs is determined by the mean free path $\lambda$ of SE for inelastic scattering, which mainly involves conduction electrons. The $\lambda$ is about $1 \mathrm{~nm}$ for metals and up to $10 \mathrm{~nm}$ for insulators. With an estimated TEM sample thickness of $80 \mathrm{~nm}$, SEs emitted from both the top and bottom surfaces of the $\mathrm{SrTiO}_{3}$ sample may be collected. These results indicate that, potentially, SEM imaging of thin TEM samples may probe not only near surface atomicdisplacements and structural reconstructions but also surface potentials.

Finally, we have performed EBIC and SE-VC on hexagonal $\mathrm{ErMnO}_{3}$ crystals with stripe domains. Detailed descriptions on crystal growth and domain structures can be found elsewhere [21]. In multiferroic $\mathrm{ErMnO}_{3}$, charged domain walls either in 'tail-to- 
tail' or 'head-to-head' configurations were observed without external biases. Moreover, enhanced (suppressed) conductance was observed at 'tail-to-tail' ('head-to-head') domain walls [22]. This observation highlights an important question regarding the charge compensation mechanism for these charged domain walls, which could be technologically important for domain wall nanoelectronics.

In Fig. 6(a), a Dark-field TEM image from the cross-sectional TEM sample of $\mathrm{ErMnO}_{3}$ shows narrow P- domains near the edge of sample (i.e., the $a b$ surface of single crystal). Surprisingly during reversal, domain structures are switched below $\sim 1 \mu \mathrm{m}$ away from the $a b$ surface; dominant domains change from $\mathrm{P}+$ on the original $a b$ surface to $\mathrm{P}$ - below $\sim 1 \mu \mathrm{m}$ away from the $a b$ surface. These $\mathrm{P}+$ dominant and $\mathrm{P}$ - dominant areas are separated by 'tail-to-tail' domain walls with negative bound charges, as shown in a schematic (Fig. 6(b)). One possible mechanism to stabilize the abnormal domain structure is through charge compensation via an accumulation of oxygen vacancies, which are double-positively charged when fully ionized. A series of SE-VC images were taken from the sample in Fig. 6 (d-g). Line profiles in Fig. 6(c) show that nonlinear potential distributions emerge with positive bias applied to the $\mathrm{ErMnO}_{3}$ crystal, while constant slopes were observed for either $0 \mathrm{~V}$ or negative biases. Notably, the steepest slope occurs around 1.4 to $3 \mu \mathrm{m}$ depth from the top electrode (Fig. 6(c)), where charged domain walls are observed (Fig. 6(b)). This implies that most electric fields are drawn around charged domain walls in the depth range of 1.4 to $3 \mu \mathrm{m}$ when a slight positive bias is applied. EBIC images from this sample are shown in Fig. 7(b). EBIC line profiles taken along the $c$-axis show that most of the ehp separation occurs in the range of 1.7 $3.8 \mu \mathrm{m}$ in depth under $10 \mathrm{~V}$, a finding which is consistent with SE-VC data. It should be 
noted that ehp diffusion may broaden the EBIC signal as compared with SE-VC data. These results also show that charged domain walls in stripe domain $\mathrm{ErMnO}_{3}$ may be studied for photovoltaic applications by controlling the charged domain walls and their compensating charges.

Our study demonstrates that EBIC and SE-VC performed in TEM provides qualitative electrostatic potential distribution maps in TEM samples, which can be used as a complementary approach to off-axis electron holography. As these techniques can be performed in STEM mode using aberration-corrected microscopes, structural information obtained by direct imaging at atomic resolution can be obtained simultaneously, thereby potentially establishing better structure-property relationships.

\section{Figure Captions}

Figure 1. Schematic of an electron-beam-induced-current (EBIC) measurement and SEM voltage contrast in STEM imaging. EBIC and SEM voltage contrast acquisitions require an electrical connection to a TEM sample. EBIC, SEM, and HAADF images were acquired simultaneously.

Figure 2. EBIC measurements from a PZT thin film grown on Nb-STO substrate. (a) STEM HAADF image showing an electrical contact made in a FIB-prepared TEM sample. (b) and (c) are HAADF and EBIC images, respectively, obtained from the biased section in (a). (d) and (e) are HAADF and EBIC images, obtained from an unbiased section shown in (a). In (c) and (e), the PZT/Nb-STO interface is indicated with dotted 
line. (f) The line profiles of EBIC signals are perpendicular to the PZT/Nb-STO interface upon application of various biases (adapted from [19]).

Figure 3. Secondary-electron voltage-contrast (SE-VC). a) STEM HAADF image, b d) SE-VC images taken with external biases, $0 \mathrm{~V}, 4 \mathrm{~V}$, and $-3 \mathrm{~V}$, respectively. e) Line profiles of SE-VC obtained with various external biases. (f) Electrostatic potential profiles obtained by off-axis electron holography (adapted from [19]). Conduction band edge $\left(\mathrm{E}_{\mathrm{c}}\right)$ and valence band edge $\left(\mathrm{E}_{\mathrm{v}}\right)$ offsets are $0.4 \mathrm{eV}$ and $0.3 \mathrm{eV}$, respectively. Detailed information on band alignment can be found in the reference [19].

Figure 4. SE-VC of $\mathrm{YMn}_{2} \mathrm{O}_{5}$ nanowires. a) SEM-STEM image obtained before attaching $\mathrm{YMn}_{2} \mathrm{O}_{5}$ nanowires to a piezo-controlled $\mathrm{W}$ probe using hydrocarbon contamination induced by a focused electron beam in the TEM. b) HAADF-STEM image and c) SE-VC image after making electrical contacts. D) Line profiles of SE-VC taken along the $\mathrm{YMn}_{2} \mathrm{O}_{5}$ nanowires under various bias conditions.

Figure 5. Atomic resolution SEM imaging. (a) HAADF STEM and (b) SEM images of hexagonal $\mathrm{YMnO}_{3}$ nanowires, synthesized by the molten salt method. (c) HAADF STEM and (d) SEM images of FIB-prepared $\mathrm{SrTiO}_{3}$ crystals. Two sets of images were simultaneously acquired. Note that the FIB-damage on the surfaces of $\mathrm{SrTiO}_{3}$ sample severely affected the signal-to-noise ratio in the SEM image, whereas a high-quality SEM image was obtained from an FIB-damage-free $\mathrm{YMnO}_{3}$ nanowire sample. 
Figure 6. (a) Dark-field TEM image of hexagonal $\mathrm{ErMnO}_{3}$ with stripe domains, viewed along the $a$-axis. Stripe domains are visible; near the edge ( $a b$ surface of crystal) of the sample, $\mathrm{P}+$ domains are dominant, but below $1 \mu \mathrm{m}$ away from the edge, $\mathrm{P}$ - domains are dominant, as shown in (b) the domain schematic within the red box. In (b), the negative bound charges and oxygen vacancies are indicated with "-." and " $V_{o}^{++}$", respectively, at the tail-to-tail domain walls. (c) Line profiles of SEM intensities from the arrow are indicated in (d-h) various SEM images, simultaneously taken with (a), under various external biases.

Figure 7. (a) HAADF image of stripe-domain $\mathrm{ErMnO}_{3}$. (b) EBIC images from the area indicated in (a) with an external bias of $0 \mathrm{~V}, 5 \mathrm{~V}$, and $10 \mathrm{~V}$, respectively. (c) Line profiles of EBIC images along the $c$-axis. Positive current flows along the $c$-axis.

\section{Acknowledgements}

This work was supported by the Materials Science and Engineering Divisions, Office of Basic Energy Sciences of the U.S. Department of Energy under Contract No.

DESC0012704. TEM sample preparation using FIB was performed at the Center for Functional Nanomaterials, Brookhaven National Laboratory. The work at Yale University was supported by NSF MRSEC Grants No. DMR 119826 (CRISP) and No. DMR 1309868 and FAME. S.-W.C. is funded by the Gordon and Betty Moore Foundation's EPiQs Initiative through Grant No. GBMF4413 to the Rutgers Center for Emergent Materials. 


\section{References}

[1] E. Volkl, L. F. Allard, and D. C. Joy, Introduction to Electron Holography (Kluwer Academic / Plenum Publishers, New York, 1999).

[2] M. R. McCartney and D. J. Smith, Annu. Rev. Mater. Res. 37, 729 (2007).

[3] M. J. Polking, M.-G. Han, A. Yourdkhani, V. Petkov, C. F. Kisielowski, V. V

Volkov, Y. Zhu, G. Caruntu, a P. Alivisatos, and R. Ramesh, Nat. Mater. 11, 700 (2012).

[4] M. Lehmann and H. Lichte, Microsc. Anal. 8, 447 (2002).

[5] Y. Yao, C. Li, Z. L. Huo, M. Liu, C. X. Zhu, C. Z. Gu, X. F. Duan, Y. G. Wang, L. Gu, and R. C. Yu, Nat. Commun. 4, 2764 (2013).

[6] F. Ubaldi, G. Pozzi, T. Kasama, M. R. McCartney, S. B. Newcomb, and R. E. Dunin-Borkowski, J. Phys. Conf. Ser. 209, 12064 (2010).

[7] J. I. Hanoka and R. O. Bell, Annu. Rev. Mater. Res. 11, 353 (1981).

[8] R. Rosenkranz, J. Mater. Sci. Mater. Electron. 22, 1523 (2011).

[9] H. J. Leamy, J. Appl. Phys. 53, R51 (1982).

[10] S. L. Elliott, R. F. Broom, and C. J. Humphreys, J. Appl. Phys. 91, 9116 (2002).

[11] G. C. Perreault and D. G. Ast, J. Phys. E. 21, 1175 (1988).

[12] C. Parish, D. Batchelor, C. Progl, and P. Russell, Microsc. Anal. 21, 11 (2007).

[13] T. G. Sparrow and U. Valdrèg, Philos. Mag. 36, 1517 (1977).

[14] S. Tanaka, H. Tanaka, T. Kawasaki, M. Ichihashi, T. Tanji, K. Arafune, Y. Ohshita, and M. Yamaguchi, J. Mater. Sci. Mater. Electron. 19, 324 (2008).

[15] M.-G. Han, Y. Zhu, K. Sasaki, T. Kato, C. a. J. Fisher, and T. Hirayama, Solid. State. Electron. 54, 777 (2010). 
[16] Ephemeron Labs, Inc., https://www.ephemeron-labs.com

[17] Y. Zhu, H. Inada, K. Nakamura, and J. Wall, Nat. Mater. 8, 808 (2009).

[18] J. Ciston, H. G. Brown, a. J. D’Alfonso, P. Koirala, C. Ophus, Y. Lin, Y. Suzuki, H. Inada, Y. Zhu, L. J. Allen, and L. D. Marks, Nat. Commun. 6, 7358 (2015).

[19] M.-G. Han, M. S. J. Marshall, L. Wu, M. a. Schofield, T. Aoki, R. Twesten, J. Hoffman, F. J. Walker, C. H. Ahn, and Y. Zhu, Nat. Commun. 5, 1 (2014).

[20] J. M. Patete, J. Han, A. L. Tiano, H. Liu, M. Han, J. W. Simonson, Y. Li, A. C. Santulli, M. C. Aronson, A. I. Frenkel, Y. Zhu, and S. S. Wong, J. Phys. Chem. C 118, 21695 (2014).

[21] X. Wang, M. Mostovoy, M. G. Han, Y. Horibe, T. Aoki, Y. Zhu, and S.-W. Cheong, Phys. Rev. Lett. 112, 247601 (2014).

[22] D. Meier, J. Seidel, a Cano, K. Delaney, Y. Kumagai, M. Mostovoy, N. a Spaldin, R. Ramesh, and M. Fiebig, Nat. Mater. 11, 284 (2012). 


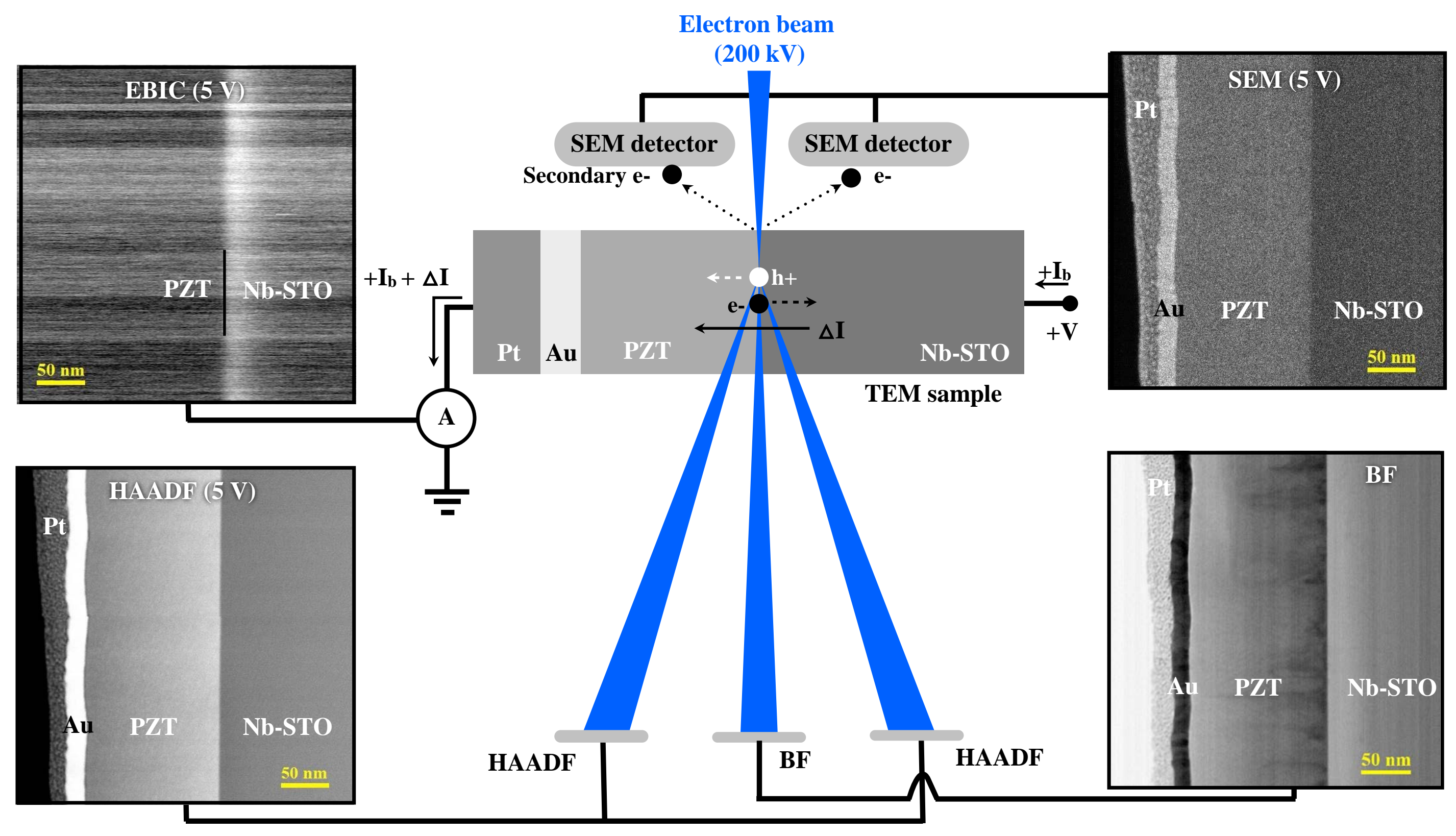

Figure 1. Schematic of an electron-beam-induced-current (EBIC) measurement and SEM voltage contrast in STEM imaging. EBIC and SEM voltage contrast acquisitions require an electrical connection to a TEM sample. EBIC, SEM, and HAADF images were acquired simultaneously. 


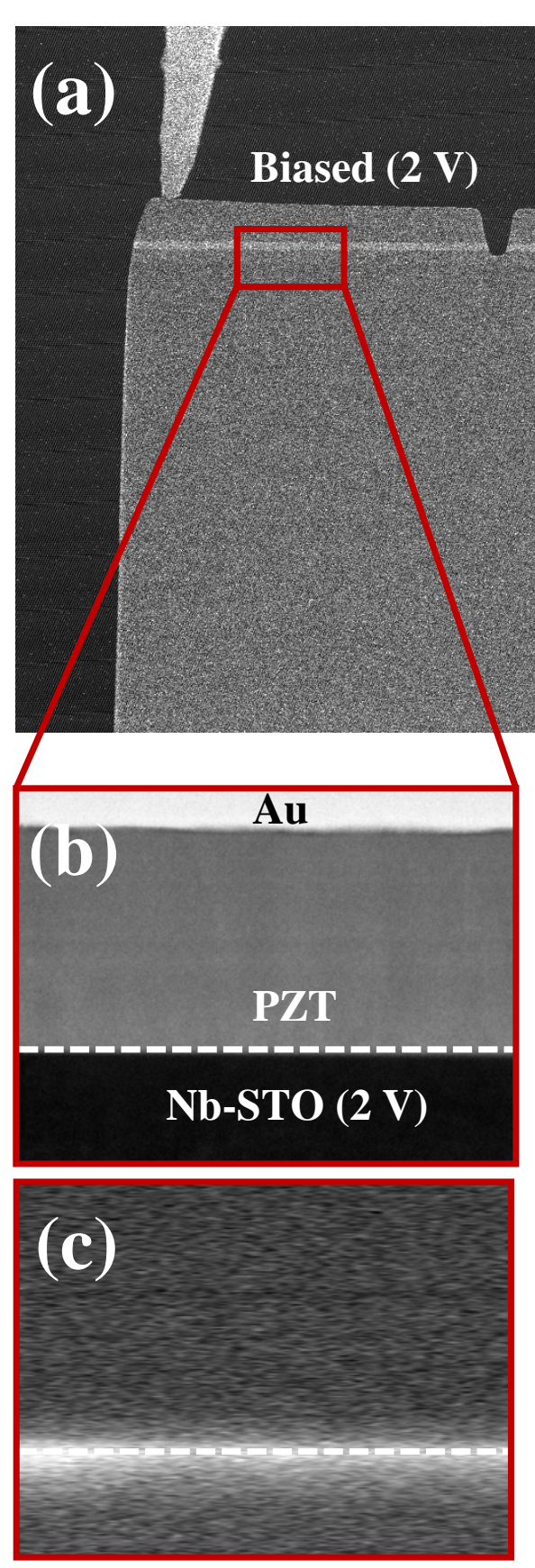

Biased (2 V)

\section{Unbiased}
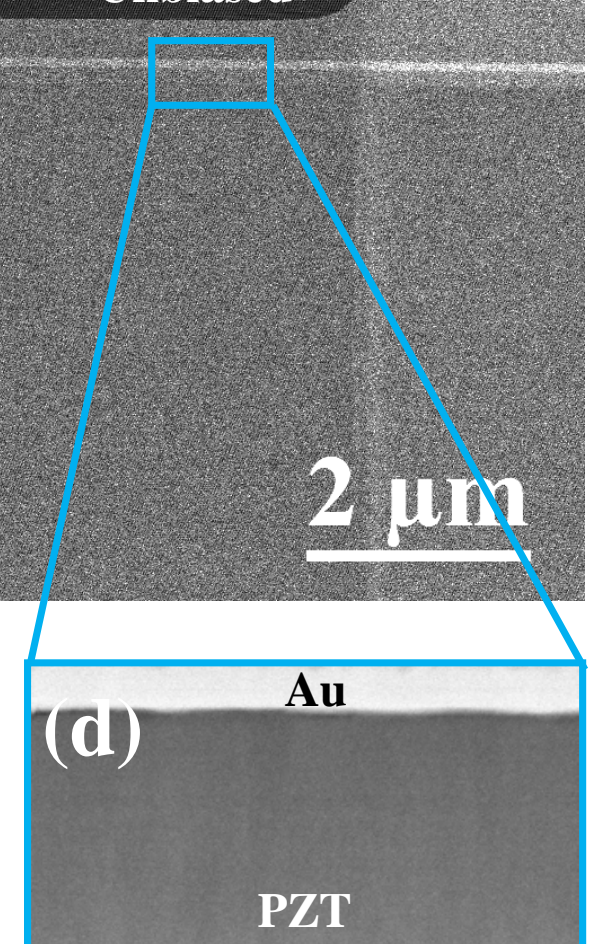

\section{$\mathrm{Nb-STO}$ (ground)}

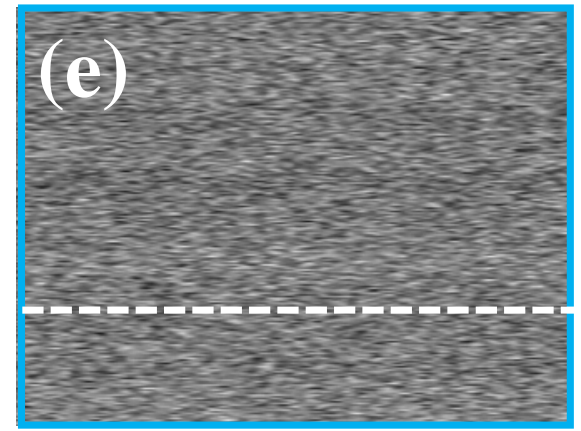

Unbiased (f)
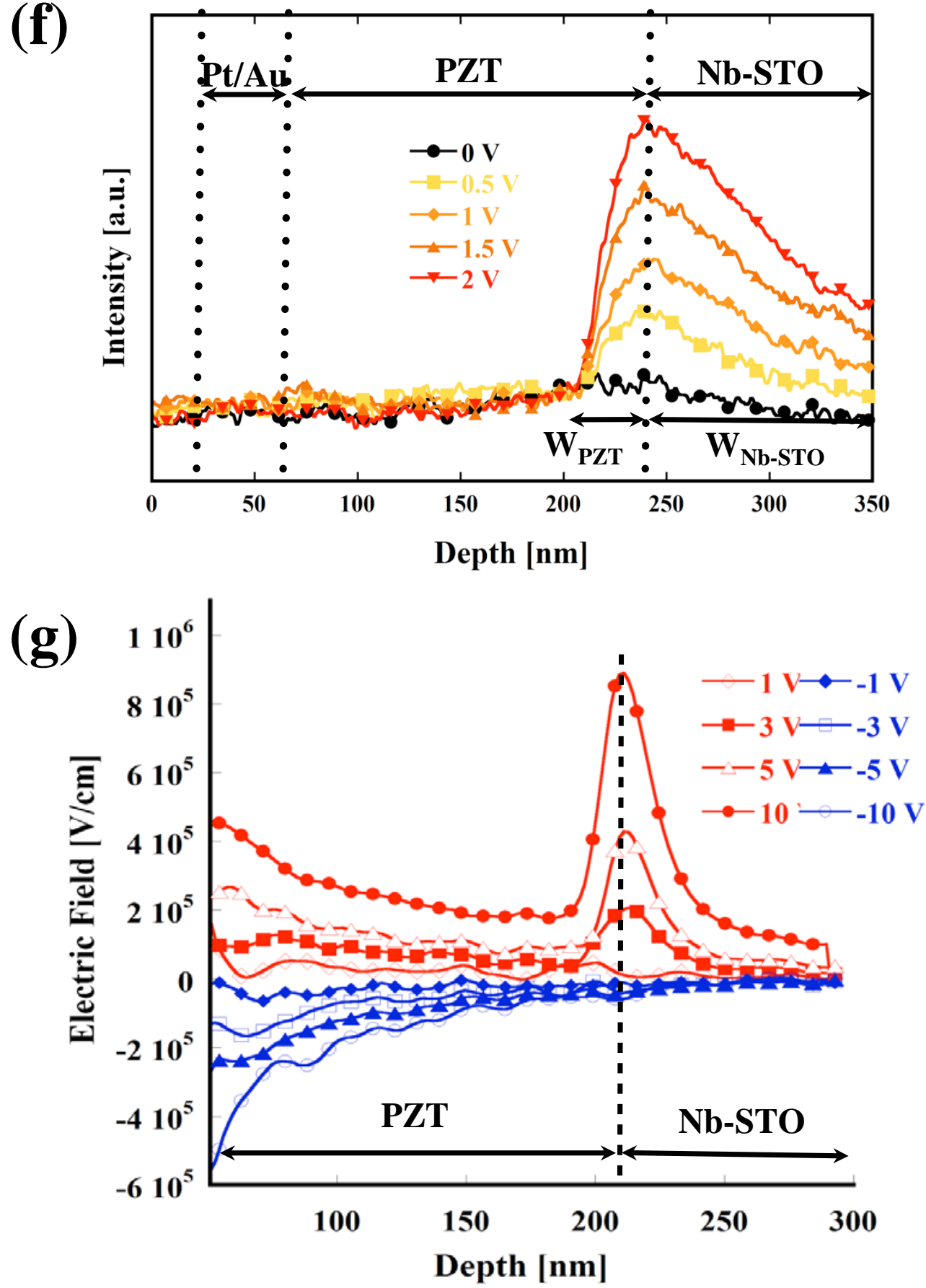

Figure 2. EBIC measurements from a PZT thin film grown on Nb-STO substrate. (a) STEM HAADF image showing an electrical contact made in a FIB-prepared TEM sample. (b) and (c) are HAADF and EBIC images, respectively, obtained from the biased section in (a). (d) and (e) are HAADF and EBIC images, obtained from an unbiased section shown in (a). In (c) and (e), the PZT/Nb-STO interface is indicated with dotted line. (f) The line profiles of EBIC signals are perpendicular to the PZT/Nb-STO interface upon application of various biases (adapted from [19]). 

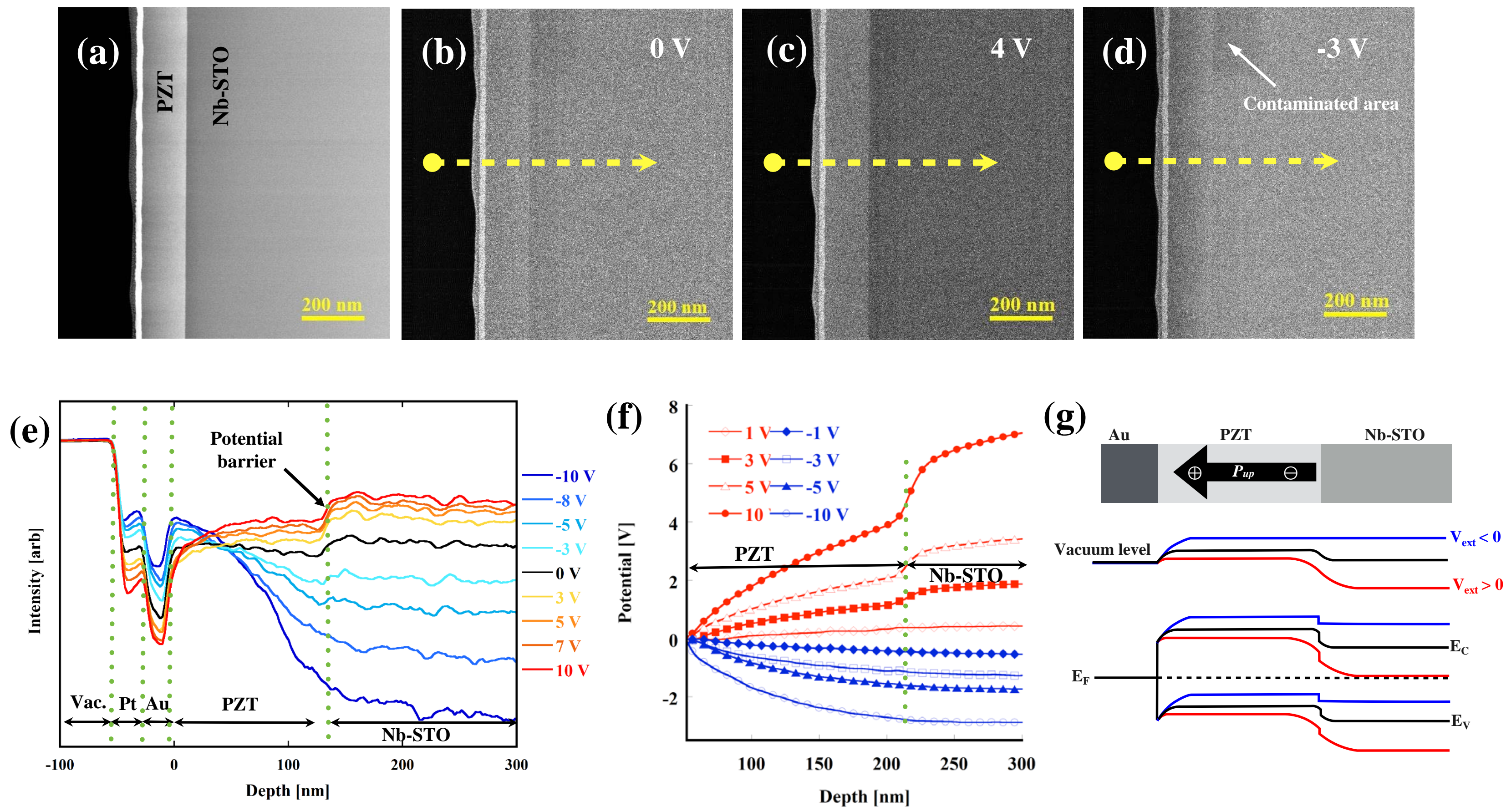

Figure 3. Secondary-electron voltage-contrast (SE-VC). a) STEM HAADF image, b d) SE-VC images taken with external biases, 0 V, $4 \mathrm{~V}$, and $-3 \mathrm{~V}$, respectively. e) Line profiles of SE-VC obtained with various external biases. (f) Electrostatic potential profiles obtained by off-axis electron holography (adapted from [19]). Conduction band edge (Ec) and valence band edge (Ev) offsets are 0.4 $\mathrm{eV}$ and $0.3 \mathrm{eV}$, respectively. Detailed information on band alignment can be found in the reference [19]. 
(a)

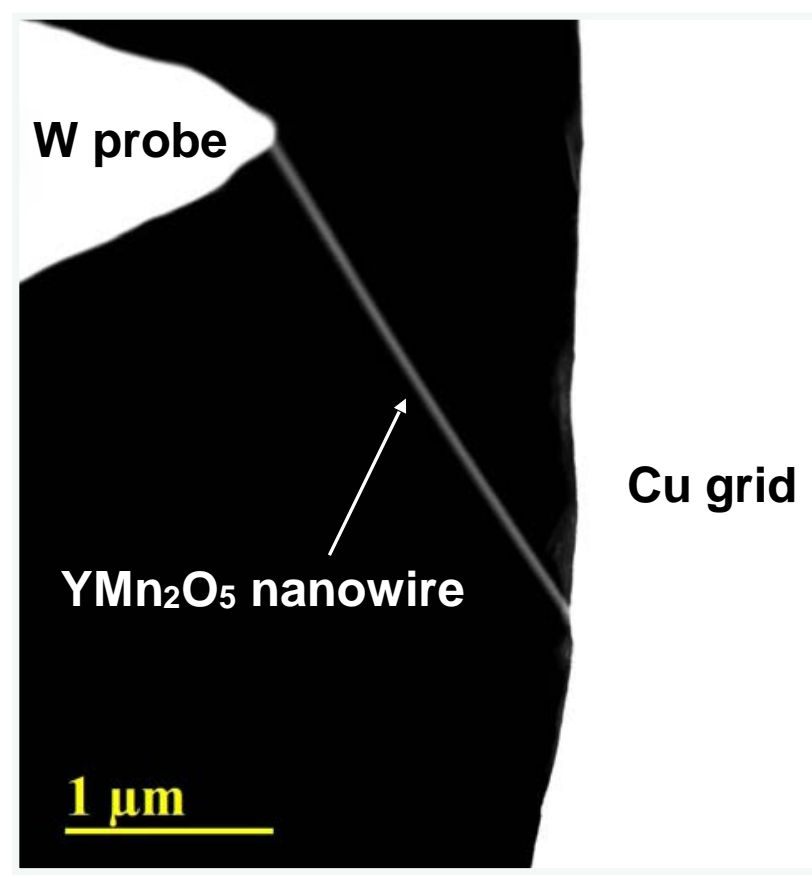

(b)

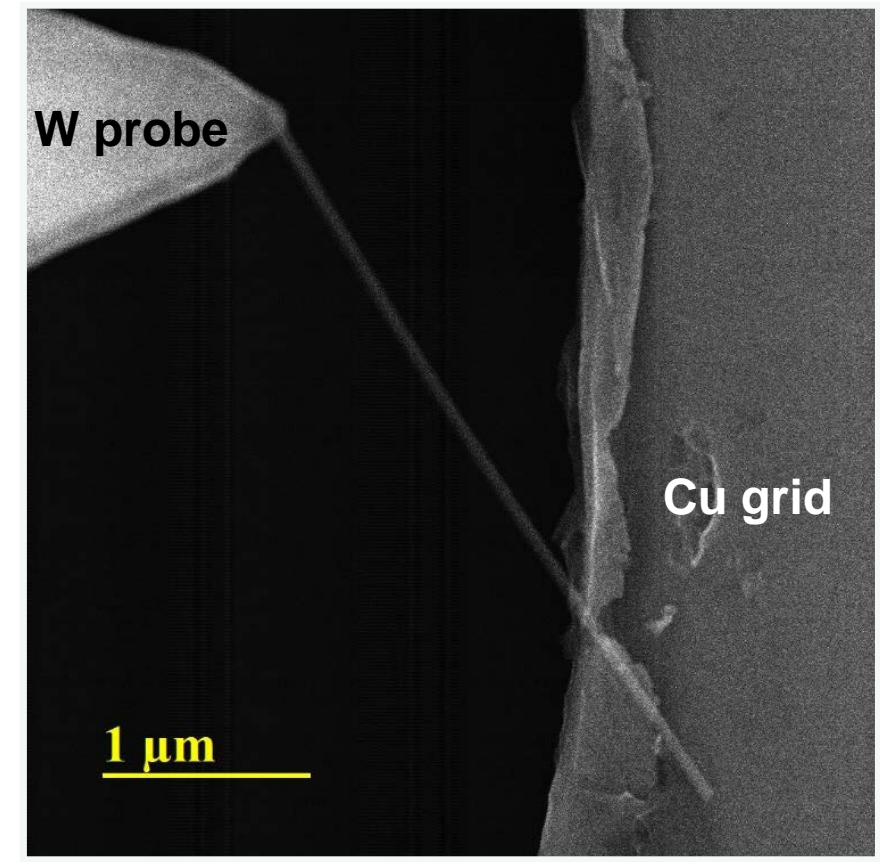

(c)

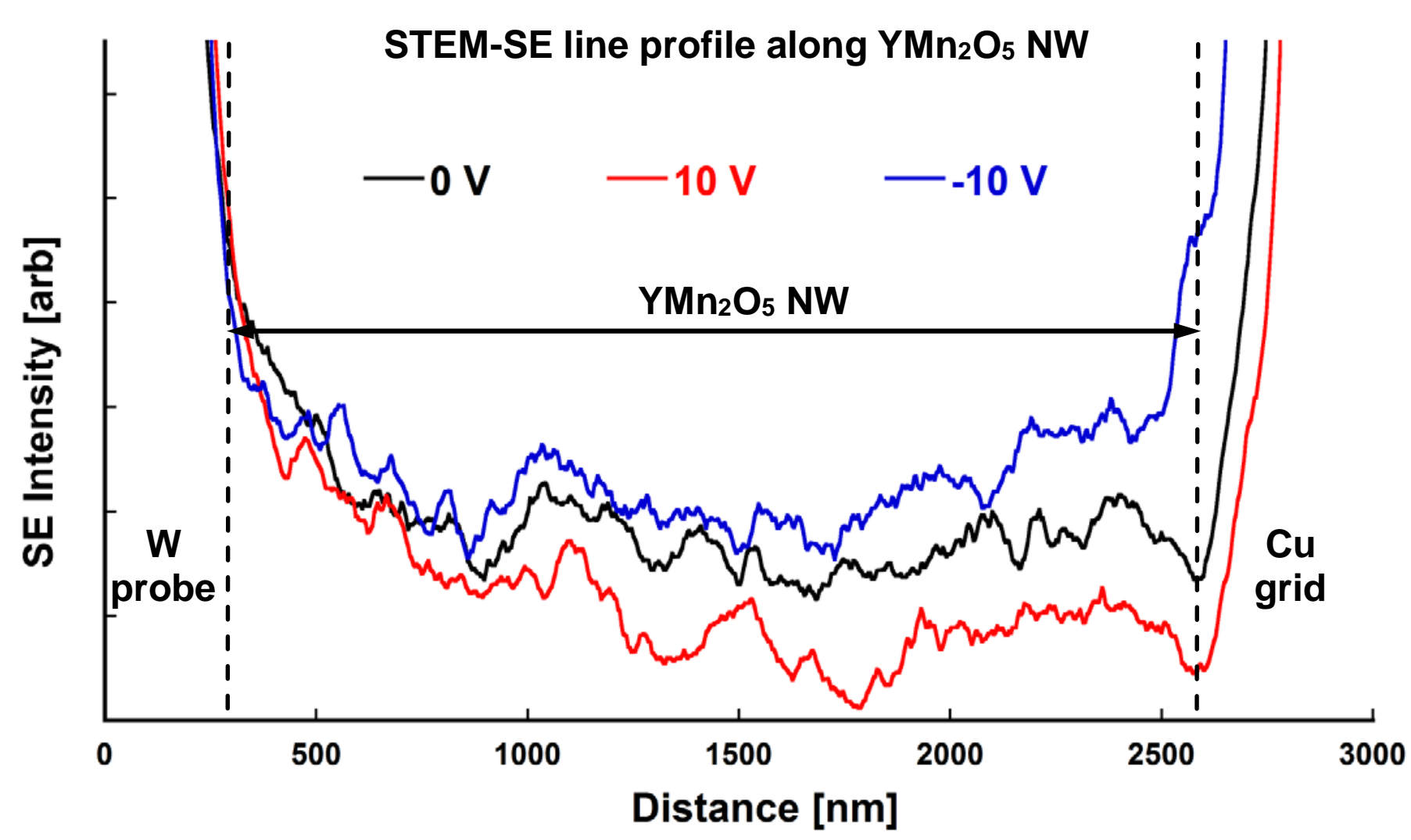

Figure 4. SE-VC of $\mathrm{YMn}_{2} \mathrm{O}_{5}$ nanowires. a) SEM-STEM image obtained before attaching $\mathrm{YMn}_{2} \mathrm{O}_{5}$ nanowires to a piezo-controlled W probe using hydrocarbon contamination induced by a focused electron beam in the TEM. b) HAADF-STEM image and c) SE-VC image after making electrical contacts. D) Line profiles of SE-VC taken along the $\mathrm{YMn}_{2} \mathrm{O}_{5}$ nanowires under various bias conditions. 


\section{(a) HAADF}

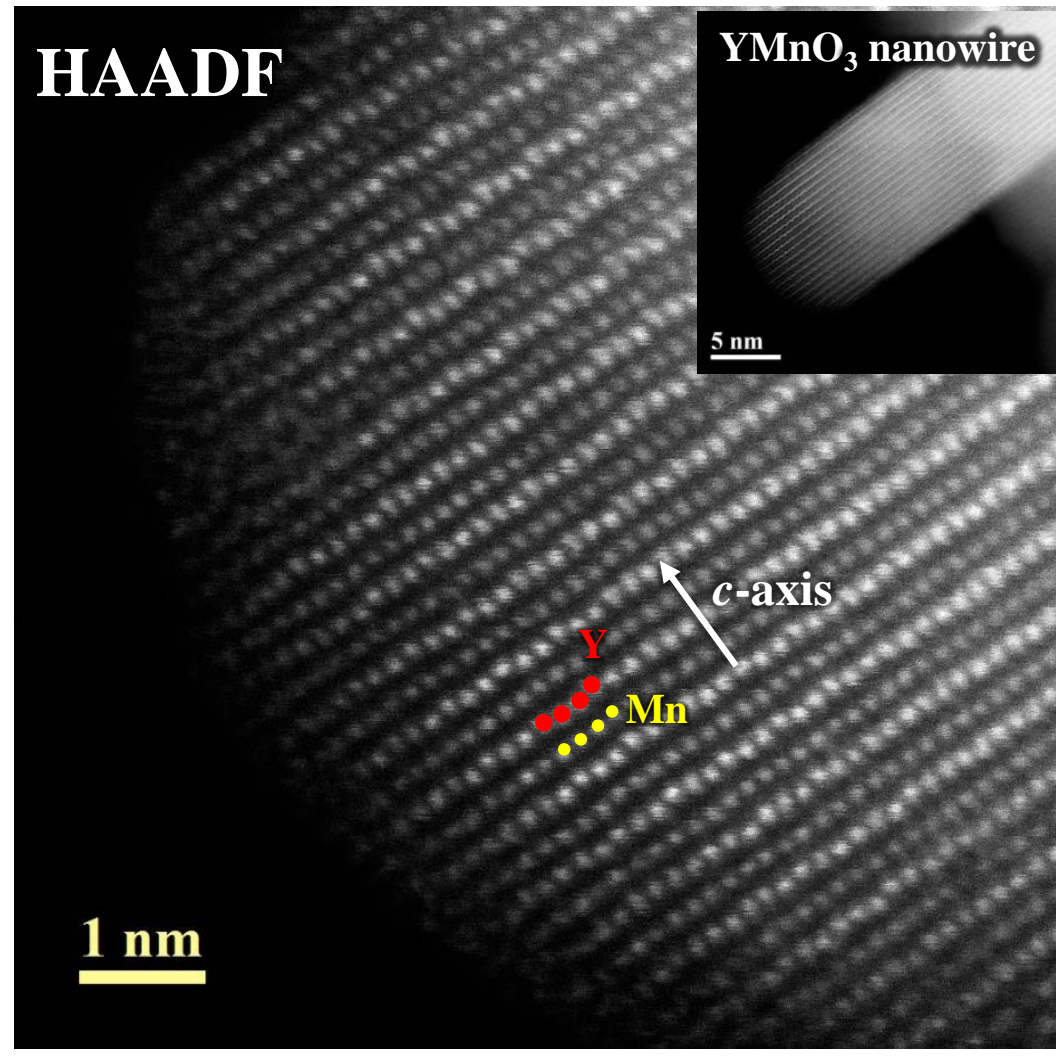



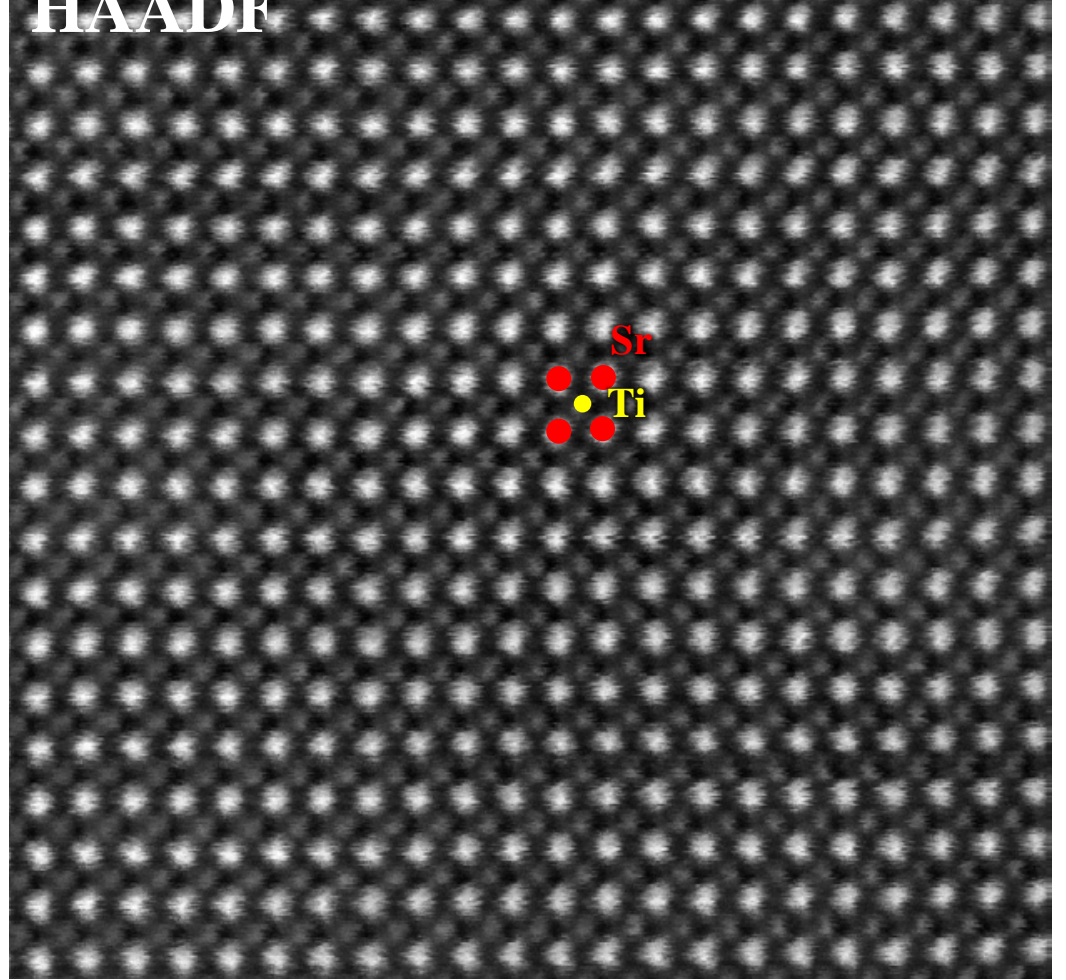

(b) SEM

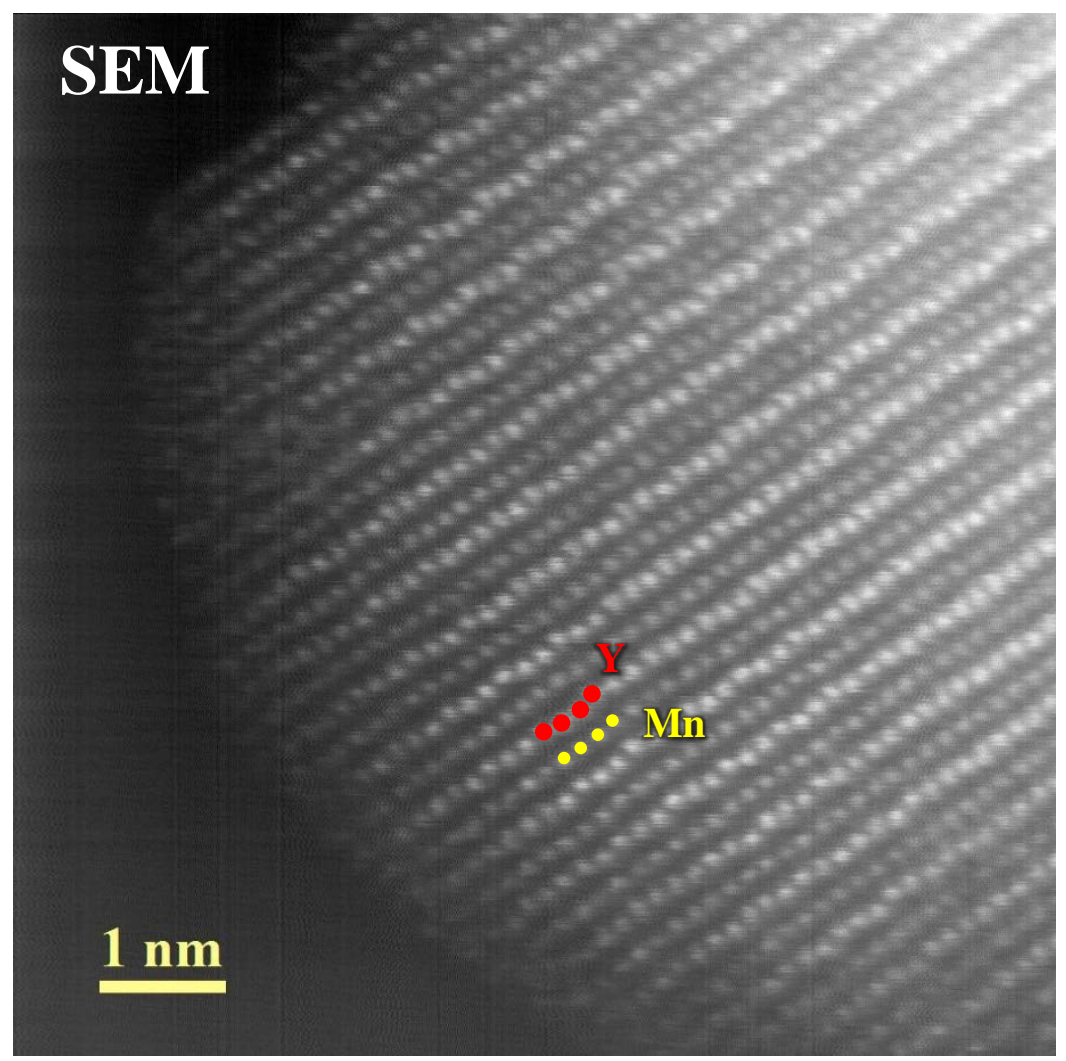

(d)

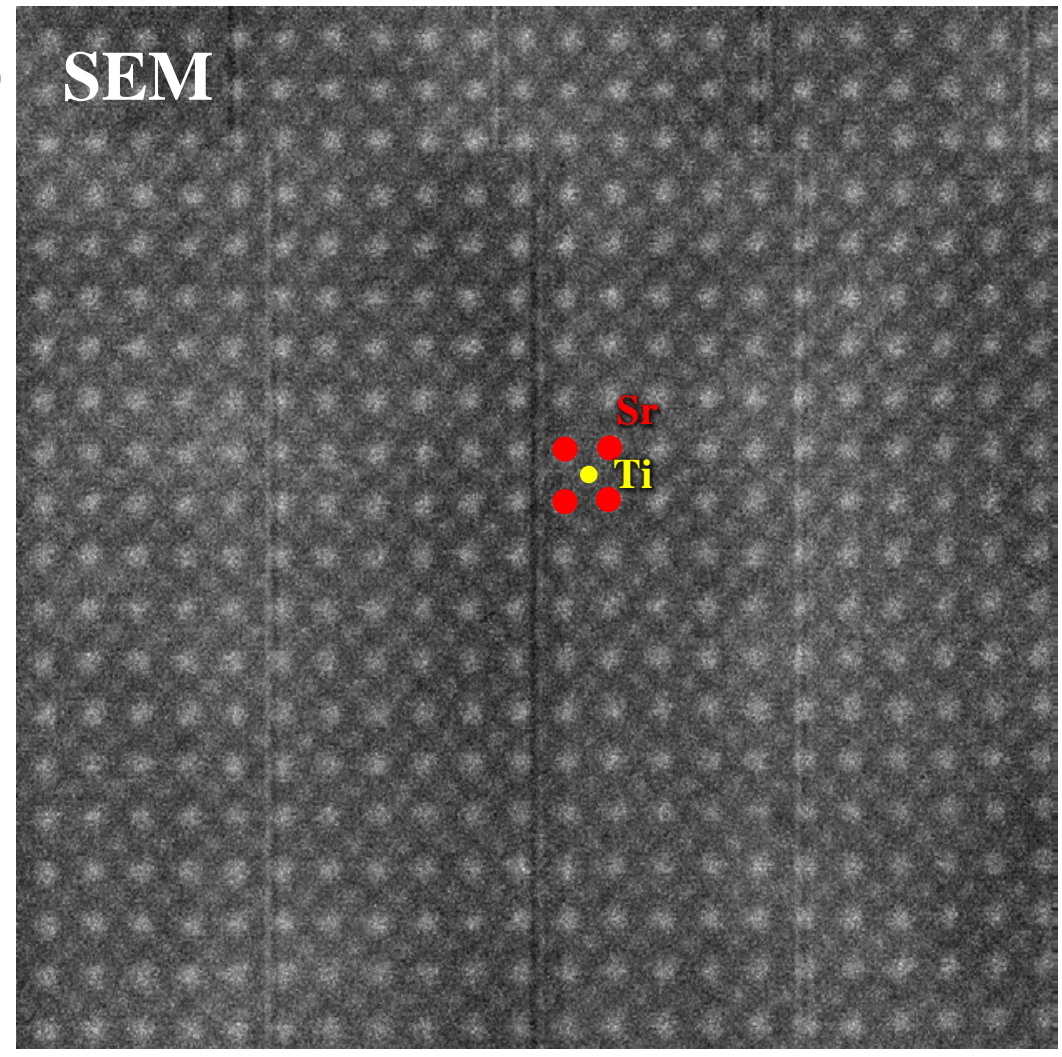

Figure 5. Atomic resolution SEM imaging. (a) HAADF STEM and (b) SEM images of hexagonal $\mathrm{YMnO}_{3}$ nanowires, synthesized by the molten salt method. (c) HAADF STEM and (d) SEM images of FIB-prepared $\mathrm{SrTiO}_{3}$ crystals. Two sets of images were simultaneously acquired. Note that the FIB-damage on the surfaces of $\mathrm{SrTiO}_{3}$ sample severely affected the signal-to-noise ratio in the SEM image, whereas a high-quality SEM image was obtained from an FIB-damage-free $\mathrm{YMnO}_{3}$ nanowire sample. 
(a)

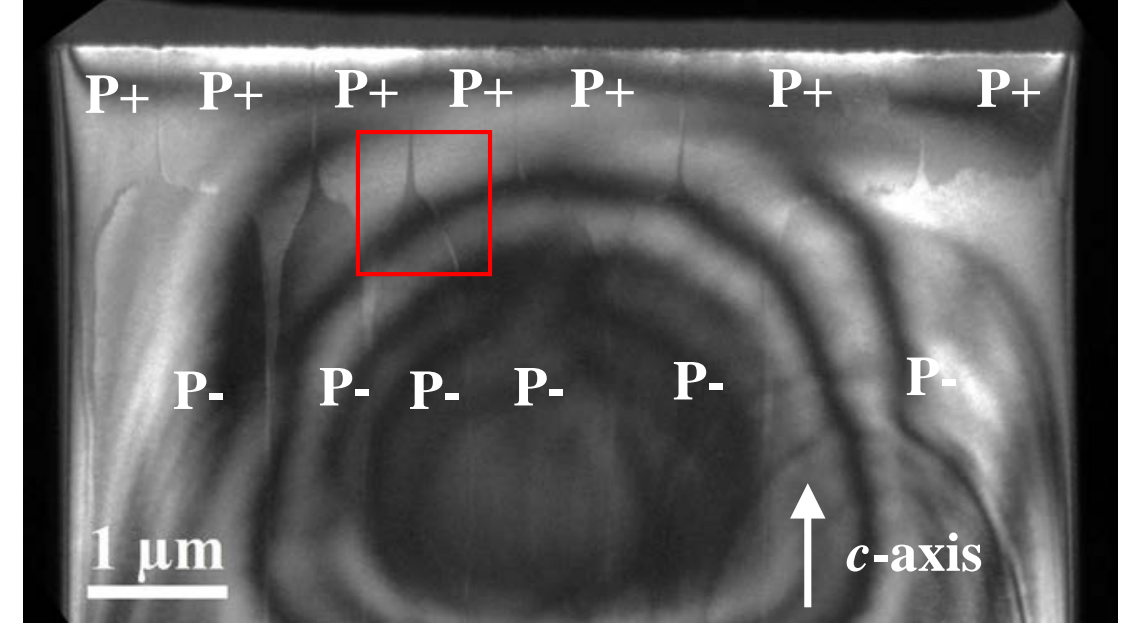

(b)

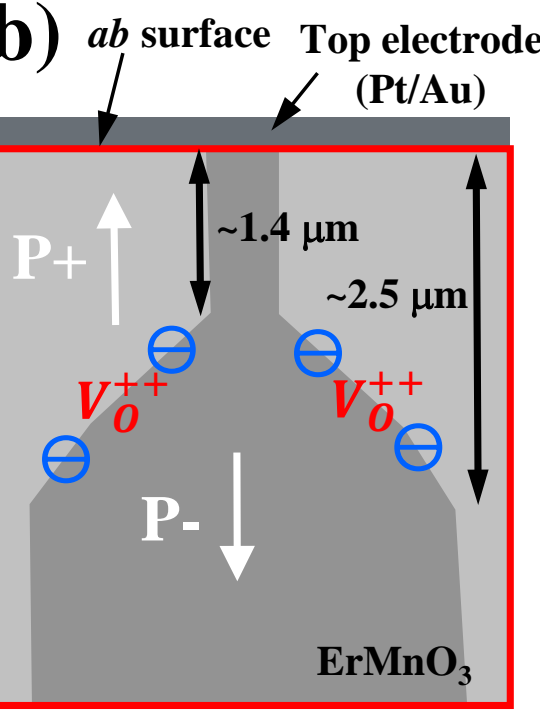

(c)
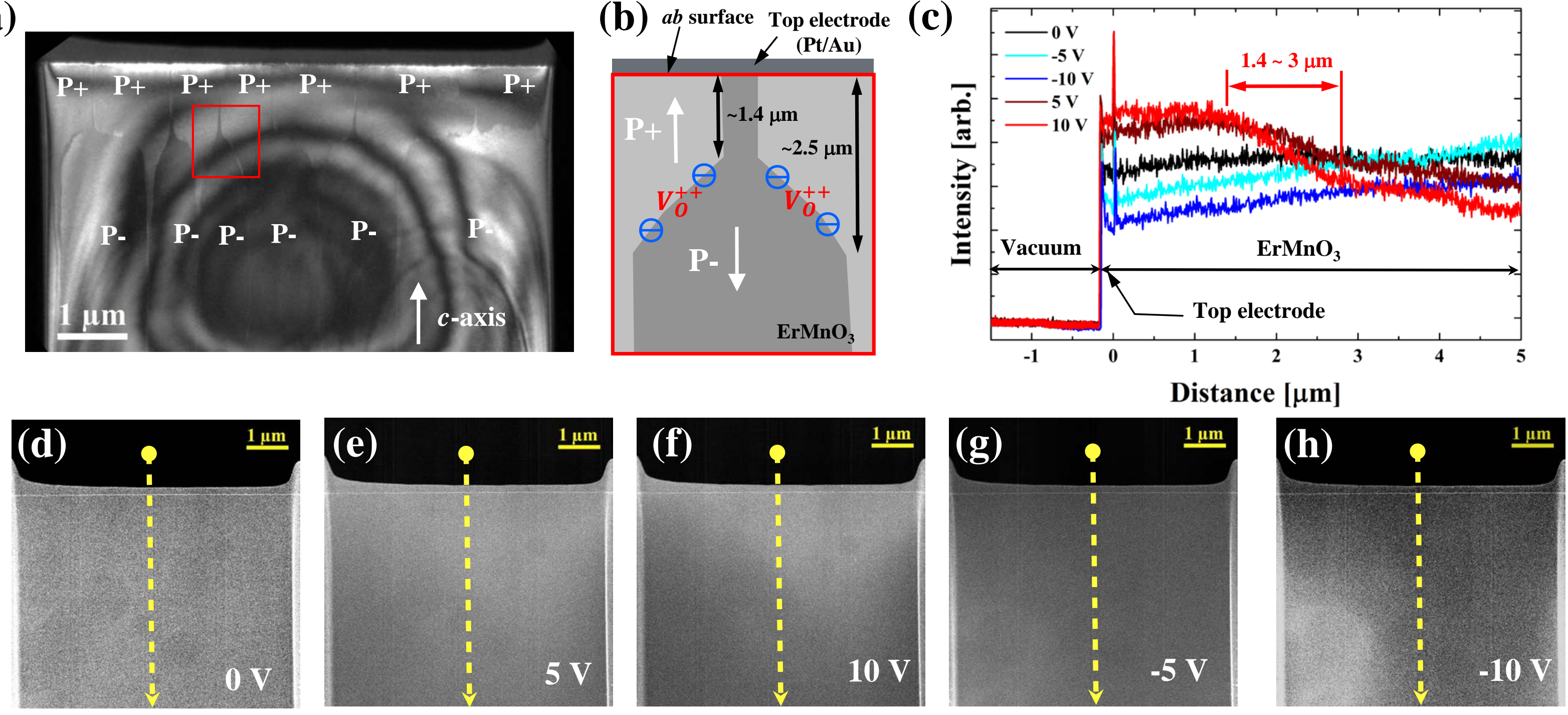

Figure 6. (a) Dark-field TEM image of hexagonal $\mathrm{ErMnO}_{3}$ with stripe domains, viewed along the $a$-axis. Stripe domains are visible; near the edge ( $a b$ surface of crystal) of the sample, $\mathrm{P}+$ domains are dominant, but below $1 \mu \mathrm{m}$ away from the edge, $\mathrm{P}$ - domains are dominant, as shown in (b) the domain schematic within the red box. In (b), the negative bound charges and oxygen vacancies are indicated with "-" and " $V_{O}^{++}$", respectively, at the tail-to-tail domain walls. (c) Line profiles of SEM intensities from the arrow are indicated in (d-h) various SEM images, simultaneously taken with (a), under various external biases. 
(a)
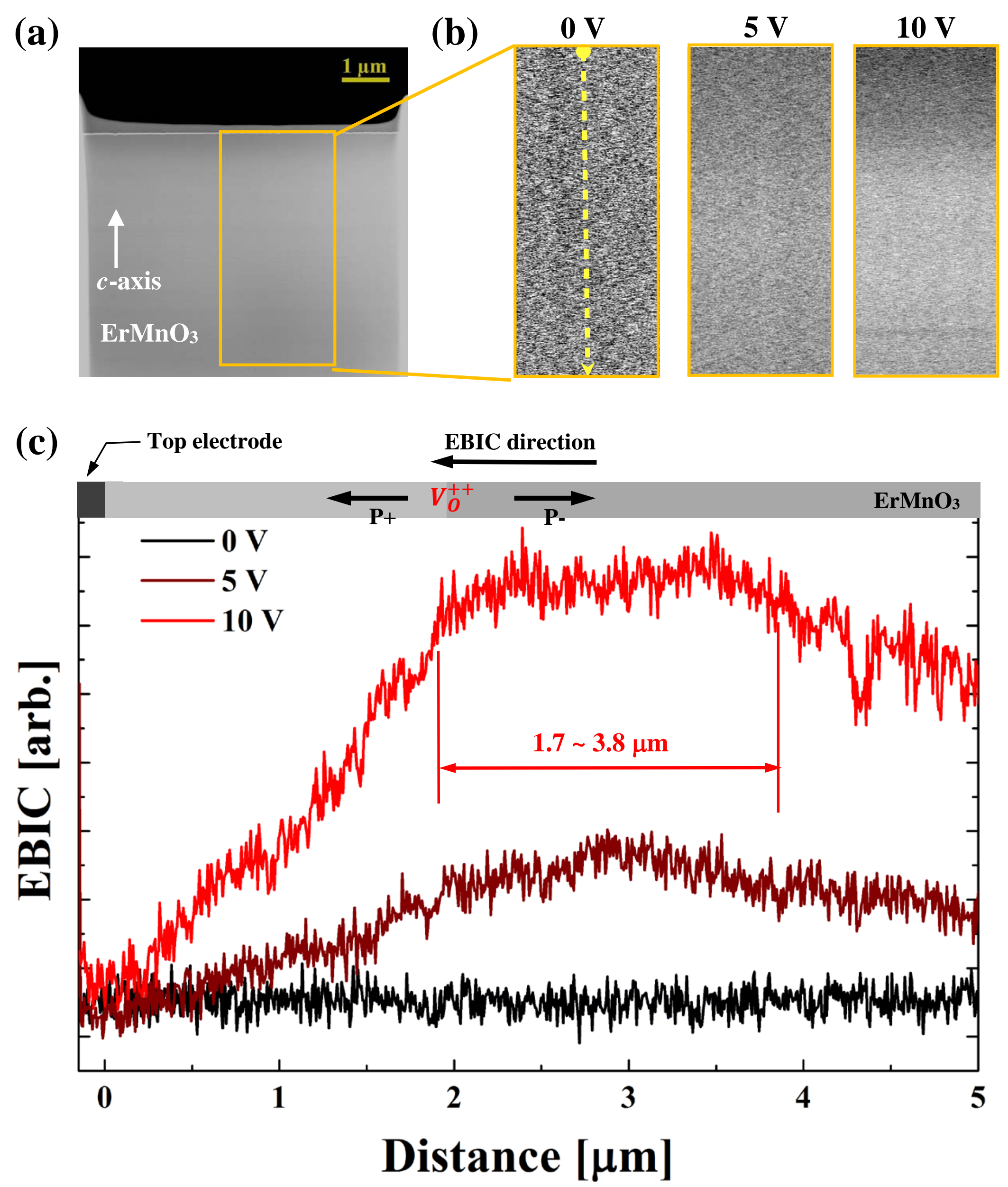

Figure 7. (a) HAADF image of stripe-domain $\mathrm{ErMnO}_{3}$. (b) EBIC images from the area indicated in (a) with an external bias of $0 \mathrm{~V}, 5$ $\mathrm{V}$, and $10 \mathrm{~V}$, respectively. (c) Line profiles of EBIC images along the $c$-axis. Positive current flows along the $c$-axis. 